\title{
Quantitative Evaluation of Bone Loss around Carious and Restored Teeth: A CBCT Study
}

\author{
Authors \\ Dr Maya S. Indurkar ${ }^{1}$, Dr Ketaki S. Dhumal ${ }^{2}$, Dr Renu Verma ${ }^{3}$ \\ ${ }^{1}$ HOD Dept of Periodontology, ${ }^{2} 3^{\text {rd }}$ Year Post Graduate Student, ${ }^{3}$ MDS Periodontology \\ Government Dental College and Hospital, Aurangabad
}

\begin{abstract}
:
Background: The teeth with proximal caries and proximal overhanging restorations lead to the food lodgement, provide a niche for plaque accumulation and increase the local factors for gingival inflammation and periodontal bone loss. So the present study aims to evaluate the amount of bone loss around such teeth.

Aim: To evaluate the amount of bone loss around teeth with caries, restoration and root canal treated teeth.

Material and Methods: 100 CBCTs of patients within the age range of 20-70 years will be included in the study. The bone loss will be assessed by direct measurement of the distance between the cementoenamel junction (CEJ) and the most coronal location of the bone margin adjacent to a clearly visible ligament space which was defined as the alveolar bone level (BL); in case of horizontal bone loss. Bone loss will considered to be present when the CEJ to alveolar bone distance is equal to $2.0 \mathrm{~mm}$ or more.

Results: The present study demonstrated that there is a statistically significant association between proximal caries, defective dental restorations and bone loss. The bone loss was found to be maximum around tooth with defective restorations followed by tooth with proximal caries; while endodontically treated teeth showed bone loss around them but the association was insignificant.
\end{abstract}

\section{Introduction}

Dental plaque is the primary initiating factor in chronic gingivitis and destructive forms of periodontal diseases. Hence it is reasonable to postulate that local factors favouring plaque accumulation and interfering with proper oral hygiene measures may play a significant role in potentiating the effect of plaque in the pathogenesis of these diseases.

Several local predisposing factors have been implicated in the etiology of chronic periodontal inflammation. Among these local factors there is significant evidence of proximal caries and incriminating flawed dental restorations in the loss of periodontal support. Unrestored caries on smooth tooth surfaces are local plaque-retention sites and may therefore be regarded as potential etiologic factors in periodontal diseases. ${ }^{1}$

Root canal treatment and periodontal bone loss The periodontium and the dental pulp are connected through different anatomic structures including the lateral and accessory canals, the apical foramina or exposed dentinal tubules after periodontal therapy. Furthermore, most of the bacteria identified in infected root canals were also found in periodontal lesions. An endodontic 
infection may exist in a non-root canal-treated, non-vital or in a root canal-treated tooth. The bacterial challenge caused by these affected teeth may lead to an inflammatory response in the adjacent periodontal tissues resulting in periodontal bone loss. ${ }^{2}$

CBCT over Conventional radiography

The chief limitation of conventional intraoral and panoramic imaging for common dentoalveolar diseases is the problem of conspicuity, which is largely the result of the representation of a 3D structure depicted by a two-dimensional (2D) image. This limitation is true for caries and periodontal and endodontic applications.

The detection of proximal and occlusal surface caries by conventional intraoral 2D methods has demonstrated only low-to-moderate sensitivity but slightly better specificity and high observer variability. Previous imaging methods for caries detection have met with limited success and dubious clinical applications. CBCT imaging appears to be the best prospect for improving the detection and depth assessment of caries in approximal and occlusal lesions and also for periodontal bone loss. ${ }^{3}$

Therefore the present study was undertaken to assess the relationship between the periodontal bone loss and untreated caries lesions, defective dental restorations affecting the approximal surfaces of teeth and endodontically treated teeth using CBCT.

Aim: To evaluate the amount of bone loss around teeth using CBCT with the following

i) Proximal caries

ii) Defective proximal dental restoration

iii) Root canal treated teeth.

\section{Subjects and Methods}

The study was carried out at Government dental college and hospital, Aurangabad. 100 CBCTs of patients; within the age range of 20-65 years were included in the study. The bone loss was assessed by direct measurement of the distance between the cementoenamel junction (CEJ) and the most coronal location of the bone margin adjacent to a clearly visible ligament space which was defined as the alveolar bone level (BL); in case of horizontal bone loss. At sites where angular bony defects were present the depth of the defect was derived from the distance of the alveolar crest (AC) and the most apical part of the defect. Bone loss was considered to be present when the CEJ to alveolar bone distance is equal to $2.0 \mathrm{~mm}$ or more. 1) Bone loss around tooth with proximal carious lesion and also the site at which the lesion is present was noted.

The following CBCT shows measurement of bone loss around teeth with proximal caries

\section{Figure 1}

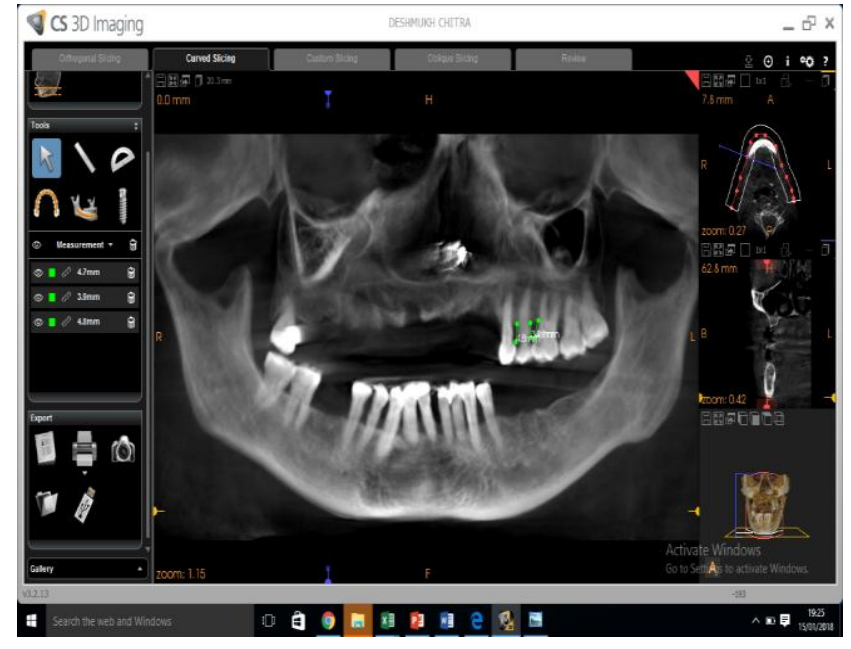

2) In case of endodontically treated teeth; the bone loss was measured on both the sides of the tooth. Amongst RC treated teeth, tooth with full crown and without full crowns are also noted.

The following cbct shows the measurement of bone loss around endodontically treated tooth

\section{Figure 2}

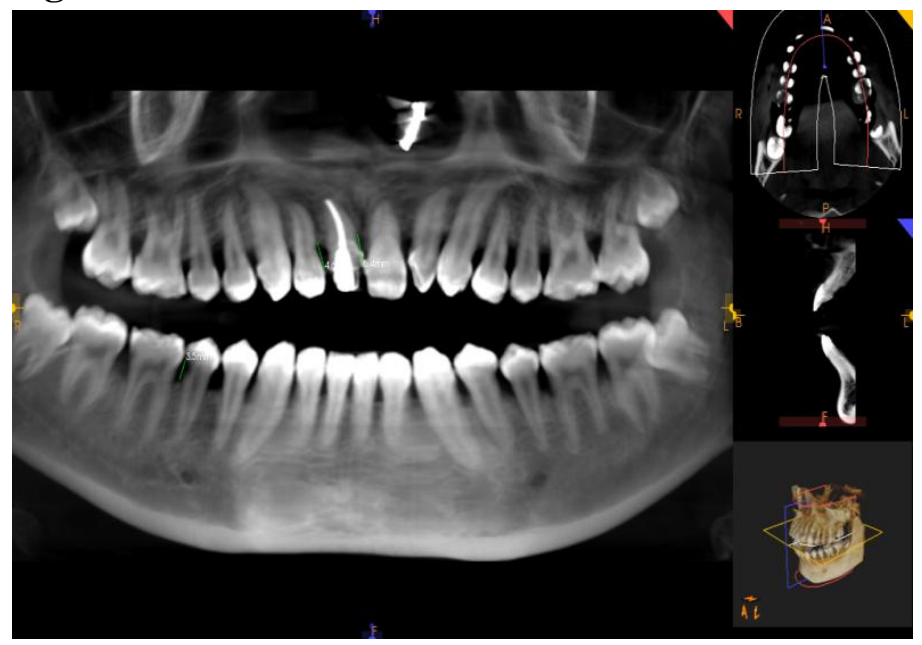


3) In case of teeth with defective dental restoration on the approximal tooth surface the bone loss around the tooth was measured.

The following cbct shows the measurement of bone loss around the tooth with defective proximal restoration

\section{Figure 3}

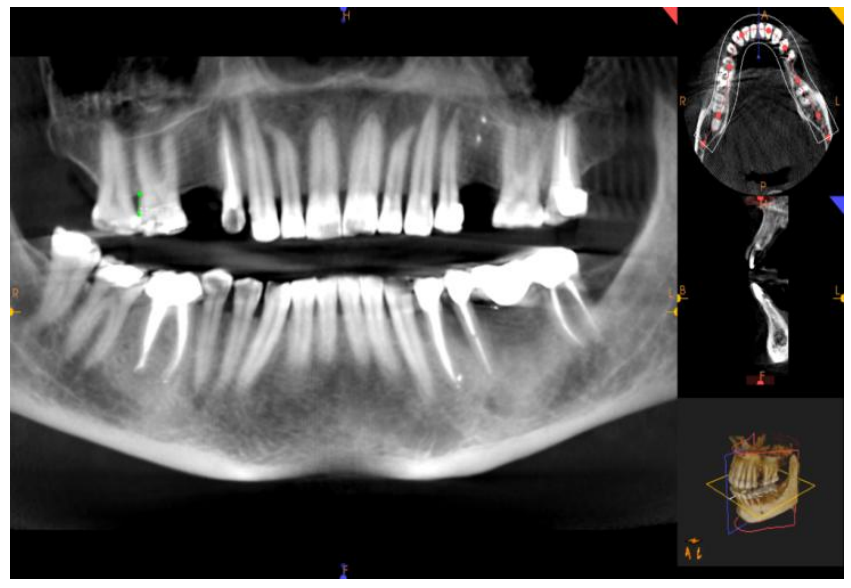

\section{Results}

A total number of 2484 teeth were assessed in 100 CBCTs. The number of approximal tooth surfaces exhibiting proximal caries lesions were 129; amongst which $57.3 \%$ were maxillary and $42.7 \%$ were mandibular. Amongst 129 teeth with proximal caries $47.2 \%$ were found on mesial side while $52.8 \%$ were found on distal side. The number of teeth with root canal treatment were 42 ; the number of teeth with defective dental restoration were 33 .

Among 129 teeth with proximal caries; about 78 teeth showed bone loss and 51 teeth showed no bone loss. Average bone loss measured around these teeth was $4.8 \mathrm{~mm}$.

Graph no.1 Graph depicting bone loss in teeth with proximal caries

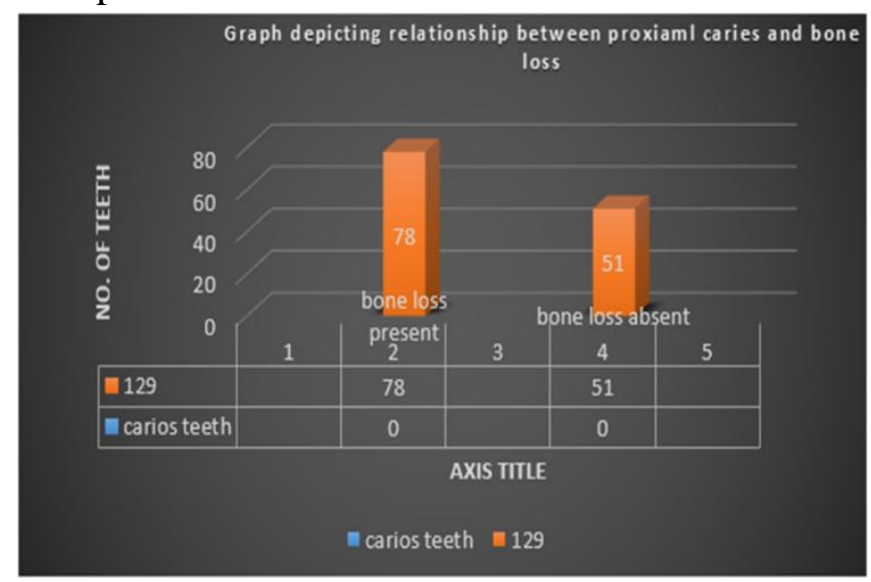

Among 42 root canal treated teeth, 17 teeth showed bone loss and 25 teeth showed no bone loss around them. Average bone loss present on the mesial side was $3.2 \mathrm{~mm}$ and distal side was $3.7 \mathrm{~mm}$. Among 42 teeth which were endodontically treated 22 teeth showed full crown and 20 teeth were without crown. 9 teeth with full crown showed bone loss while 10 teeth without them showed bone loss.

Graph no. 2 Graph depicting bone loss in teeth with endodontically treated teeth

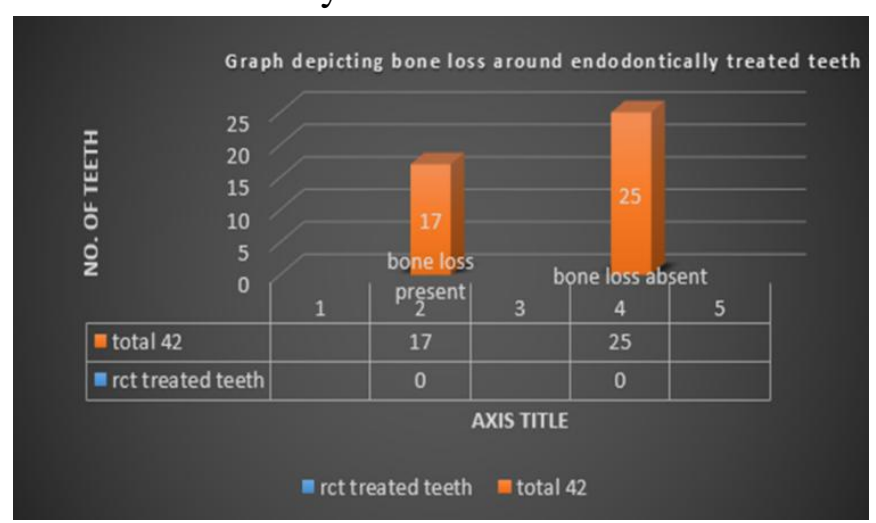

Among 33 teeth with defective dental restoration 30 teeth showed bone loss around them while 3 teeth showed no bone loss. Average bone loss around them was $5.2 \mathrm{~mm}$

Graph no. 3 Graph depicting bone loss in teeth with defective restorations.

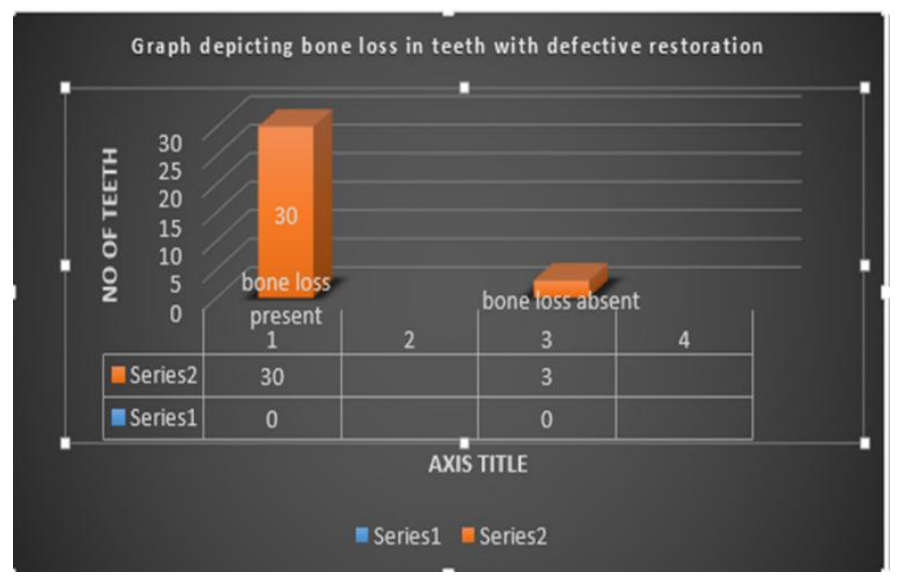

There was a statistically significant correlation between the presence of proximal caries and bone loss as well as defective restorations and bone loss by applying chi square test. While there was no correlation found between bone loss and presence of root canal treated teeth by applying chi- square test. 


\section{Discussion}

This study established a significant association between the presence of untreated manifest caries lesions, defective dental restorations, and the progression of periodontal support loss in patients. Hence, approximal tooth surfaces exhibiting these local factors are more prone to demonstrate a progressive deterioration of their periodontal support. Furthermore, the adjacent approximal surface of the neighbouring tooth also demonstrated higher loss in the periodontal support.

In the present study, association between defective dental restorations and periodontal bone loss was found to be most significant; followed by teeth with proximal caries. This highly emphasises the importance of the maintenance of proper contact and contour while restoring lesions on proximal surfaces.

There is reason to believe that the prevalence of faulty dental restorations generally is rather high. A 1990 report found that $62 \%$ of approximal restorations made at a dental school had overhanging margins. In the present material $90 \%$ of the approximal restorations examined had overhangs or secondary caries.

In a similar study; when the effect on alveolar bone height was evaluated; the presence of caries and defective and non-defective restorations at the implicated site was associated with lower alveolar bone height which was highly significant ( $\mathrm{P}<$ $0.00001)$. On the other hand, the presence of these three variables at the neighboring tooth surface also had a statistically significant effect ( $\mathrm{P}<$ 0.05 ), though of less magnitude than the effect of the variables scored at the same tooth surface. ${ }^{1}$

Dental restorations are foreign elements with a limited resemblance to the tooth form, and almost no resemblance to its composition. Dentists, in their effort to repair teeth and restore oral esthetics and function, only attempt to mimic the physical and other features of teeth, typically with less than optimal success. Overhanging margins of amalgam and other tooth restorations are local plaque-retention sites and in this regard, potential etiologic factors particularly when located subgingivally. Artificial crown margins can exert similar effects on the periodontal tissues.

It has been suggested that the margin of the final prosthesis/restoration should be placed 1 to $2 \mathrm{~mm}$ supragingivally wherever possible as locating the margins too close to the bone may cause periodontal destruction because of hindrance to plaque removal by routine oral hygiene procedures. If required, for esthetic considerations, the margin can be placed at the gingival crest or, at the most, $0.25 \mathrm{~mm}$ to $0.5 \mathrm{~mm}$ into the gingival sulcus. This ensures that the biologic width remains healthy. Therefore, the most critical factor regarding the long-term gingival health is the relationship between the supracrestal fiber attachment and margin location and the location of the base of the sulcus. If the margins are placed into the zone of the biologic width, a very important biologic principle is being violated. When the biologic width is violated, an inflammatory response results in alveolar bone resorption, increased pocket depths, increased loss of periodontal support, exacerbation of accumulation of subgingival bacteria, increased chronic inflammation, and further localized periodontal breakdown. ${ }^{4}$

Previous studies have used intraoral periapical radiographs and bitewing radiographs for the detection of caries; but the present study uses CBCT which is more accurate in the measurement of the amount of bone loss.

The present study supports the premise that the local factors such as untreated cavities, defective restorations, have a significant negative effect on periodontal health in adolescents. Young individuals having multiple sites with such local factors are to be considered at risk of developing early periodontal lesions, and should be treated accordingly.

\section{References}

1. Jasmin M. Albandar*, Yvonne A.P. Buischi/ and Per Axelsson* Caries Lesions and Dental Restorations as Predisposing 
Factors in the Progression of Periodontal diseases in Adolescents. A 3- Year Longitudinal Study. J Periodontol 1995

2. Raja Sunitha V, Pamela Emmadi, Ambalavanan Namasivayam, Ramakrishnan Thyegarajan, and Vijayalakshmi Rajaraman The periodontal - endodontic continuum: A review, J Conserv Dent 2008.

3. Donald Tyndall DDS, MSPH, PHD, Sonali Rathore, BDS, Cone beam CT diagnostic applications: Caries, periodontal bone assessment and endodontic applicatins, Den clin N Am 52 (2008)

4. Arvind Shenoy1, Nina Shenoy2, Roopa Babannavar1 Periodontl considerations determining the design and location of margins in restorative dentistry, Journal of interdisciplinary dentistry 2012. 